\title{
Clinical application of MOLLI T1* for extracellular volume calculation in healthy volunteers and aortic stenosis
}

\author{
Anish N Bhuva*, Thomas A Treibel, Arthur Nasis, Stefania Rosmini, Amna Abdel-Gadir, Heerajnarain Bulluck, \\ Charlotte Manisty, James Moon
}

From 18th Annual SCMR Scientific Sessions

Nice, France. 4-7 February 2015

\section{Background}

The calculation of the extracellular volume fraction (ECV) requires accurate quantification of myocardial and blood pool T1. Some Modified look locker inversion recovery (MOLLI) sequences provide a $\mathrm{T} 1$ and $\mathrm{T} 1^{*}$ output. T1* does not use a look locker correction, and so it is theoretically a more accurate estimation of true T1 blood $\mathrm{T} 1$ because fresh spins are flowing into the imaging plane. It is therefore recommended to use $\mathrm{T} 1$ * for the quantification of the pre- and post-contrast blood pool. The aim of this study was to investigate the effect on ECV of using $\mathrm{T} 1 *\left(\mathrm{ECV}_{\mathrm{T} 1}\right)$ rather than $\mathrm{T} 1\left(\mathrm{ECV}_{\mathrm{T} 1}\right)$ and assess accuracy, precision and bias.

\section{Methods}

57 patients with aortic stenosis (AS) (mean age $=71 \pm 10$ years, 33 female) and 25 healthy volunteers (HV) (mean age $=40 \pm 11$ years, 19 female) were recruited. 4 chamber and mid ventricular short axis (SA) T1 maps were acquired pre-contrast and 15 minute post-contrast using $5 \mathrm{~s}(3 \mathrm{~s}) 3 \mathrm{~s}$ and $4 \mathrm{~s}(1 \mathrm{~s}) 3 \mathrm{~s}(1 \mathrm{~s}) 2 \mathrm{~s}$ sequences respectively. Regions of interest (ROI) were drawn carefully to avoid blood-myocardium border and copied across series with correction only for patient movement. ECV was calculated as $\left(\Delta\left[1 / \mathrm{T} 1_{\text {myo }}\right] / \Delta\left[1 / \mathrm{T} 1_{\text {blood }}\right]\right) *$ (1-haematocrit).

\section{Results}

$\mathrm{ECV}_{\mathrm{T1}_{1}}$ was significantly lower than $\mathrm{ECV}_{\mathrm{T} 1}$ (mean 27.1 $\pm 3.4 \%$ vs $28.1 \pm 3.2 \%, \mathrm{p}<0.0001)$. $\mathrm{ECV}_{\mathrm{T} 1 *}$ showed excellent correlation with $\mathrm{ECV}_{\mathrm{T} 1}(\mathrm{R}=0.88)$ (Figure 1). Bland-Altman analysis revealed no bias or variability (Figure 2 ). There was no statistical difference in variance between groups

The Heart Hospital Imaging Centre, London, UK

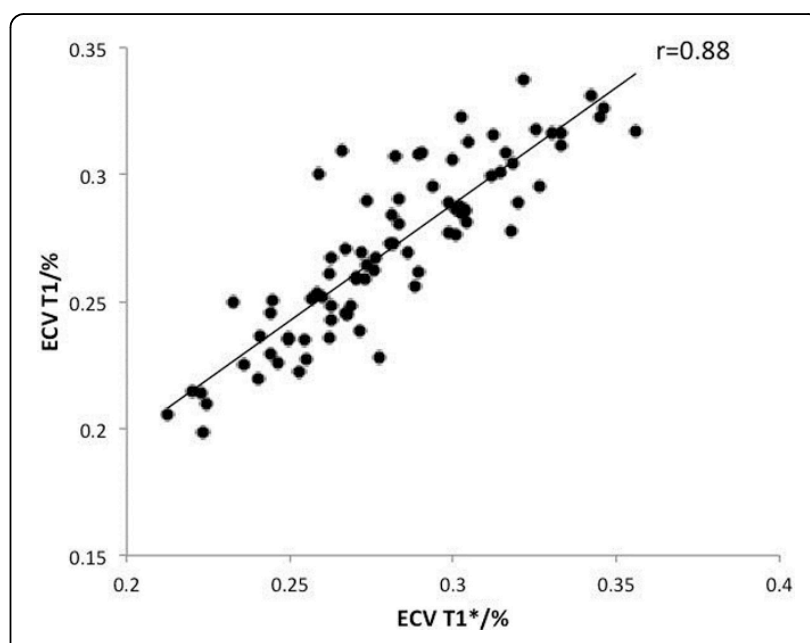

Figure 1 Excellent correlation between ECV BloodT1 and ECV BloodT1*

(F test, $\mathrm{p}=0.66$ ). In this group of subjects there was no difference in ECV between AS and HV groups using either $\mathrm{ECV}_{\mathrm{T} 1}(28.1 \pm 3.2 \%$ vs $28.2 \pm 3.4 \%)$ or $\mathrm{ECV}_{\mathrm{T} 1^{*}}(27.3 \pm 3.6 \%$ vs $26.5 \pm 3.0 \%)$.

\section{Conclusions}

ECV quantification using T1* can measure ECV across disease and normal populations, but its own normal values need to be referenced. It has similar variability, and no bias when compared to $\mathrm{ECV}$ using $\mathrm{T}_{\text {blood. }} \mathrm{ECV}_{\mathrm{T} 1^{*}}$ is therefore practically feasible and encourages further work to explore its theoretical accuracy by histological correlation.

\section{Funding}

N/A. 


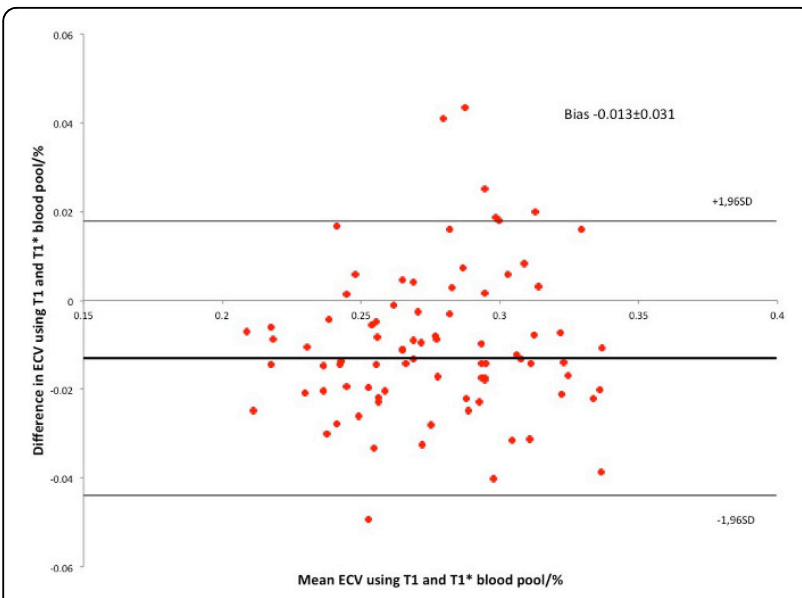

Figure 2 Little bias and variability between ECV BloodT1 and BloodT1* using Bland-Altman analysis

Published: 3 February 2015

doi:10.1186/1532-429X-17-S1-P11

Cite this article as: Bhuva et al: Clinical application of MOLLI T1* for extracellular volume calculation in healthy volunteers and aortic stenosis. Journal of Cardiovascular Magnetic Resonance 2015 17(Suppl 1): P11. 